\title{
Optimal Parameter Settings for Forward Error Correction Schemes for Multimedia Streaming over Wireless Networks
}

\author{
Claus Bauer, Wenyu Jiang, Dolby Laboratories, San Francisco, CA, USA, \{cb,wzj\}@dolby.com
}

\begin{abstract}
This paper investigates algorithms to determine an optimal choice of the FEC parameters $(n, k)$ to mitigate the effects of packet loss due to buffer overflow at a wireless base station on multimedia traffic. We develop an analytic model of the considered network scenario that takes into account the traffic arrival rates, the channel loss characteristics, the size of the buffer at the wireless access point, and the influence of the FEC parameters on the packet loss. Applying the theory of recurrent linear equations, we present a new approach to establish a closed form solution of the underlying Markov model for the buffer occupancy and verify the analytical results via simulations.
\end{abstract}

\section{INTRODUCTION}

The delivery of real-time multimedia traffic over wireless networks is expected to be an important application in future wireless networks. The perceptual quality of service as received by the user suffers from packet loss, delay, and delay jitter. Packet loss in wireless networks is due to two causes:

1) Packets might get lost or arrive corrupted at the receiver when they are transported over the lossy air medium. This can be caused by a low signal to noise ratio or collisions with packets sent from neighboring access points or nodes.

2) Packets are dropped from the queue of the wireless access point (AP) if the queue is full.

In the following, we will refer to packet losses resulting from reasons 1 and 2 as losses of type 1 and 2, respectively.

Forward Error Correction (FEC) codes have been widely researched as a method to achieve improved QoS [2], [9]. FEC codes have been shown to be very effective against packet loss provided that the losses are not too bursty [1]. A FEC block code with a parameter set $(n, k)$ takes a codeword of $k$ data packets and generates $n-k$ additional parity packets, such that a total of $n$ packets are transmitted over the network. If the sum of multimedia and FEC packets received at the receiver is at least $k$, then $k$ multimedia packets are recovered. Otherwise only the received multimedia packets are recovered.

The rapidly changing network conditions in communication systems imply a need for adaptive FEC protection schemes that dynamically change the FEC parameters $(n, k)$ depending on the current network conditions. Various adaptive FEC schemes have been proposed [3], [5], [8]. These papers make no distinction between losses of type 1 and 2 and design generic FEC algorithm for any lossy channel. The effect of FEC codes to protect against packet losses of type 1 has been investigated in [6].

In these articles, the effectiveness of a new FEC parameter set is calculated using the current loss probability of the considered traffic stream, i.e., it is assumed that the loss probability of the channel is not influenced by a change in the sending rate of the multimedia traffic due to a change in the set of FEC parameters. For losses of type 1 this assumption is roughly correct, if one assumes that the channel has enough bandwidth to send the original multimedia packets as well as the additional FEC packets at the same bit rate it was operating before changing the actual FEC parameters $(n, k)$. In contrast, for packet losses of type 2, a change of the FEC parameters leads to a change in the overall sending rate of the multimedia traffic that in turn leads to a different occupancy of the AP queue and thus influences the frequency at which losses of type 2 occur. Consequently, we see that the algorithms described in previous papers only predict the effectiveness of FEC codes correctly for losses of type 1 , but not of type 2 .

In [4], for the first time the effectiveness of FEC parameters in view of losses of type 2 was investigated. However, the continuous time model applied in [4] leads to a very complex mathematical derivation and the actual calculation of the effectiveness of the FEC parameters $(n, k)$ is computationally very expensive. The less complex, asymptotic algorithms also proposed in [4] only give valid results for a limited range of input parameters.

In this paper, we consider the problem of determining the efficiency of FEC parameters $(n, k)$ in view of losses of type 2 . In contrast to [4], we use a discrete time model that provides both a short mathematical derivation as well as an exact and computationally simple solution for all possible values of network parameters. Different from previous work quoted above, the current packet loss is not used explicitly as an input parameter for the algorithm to determine the FEC parameters. Instead, we develop a model of the network that takes explicitly into account the arrival patterns of all traffic streams in the network, the size of the buffer at the access point, and the reliability of the wireless channel.

Technically, our approach is as follows: First, we develop a Markov steady state model for the queue occupancy. Applying the theory of linear recurrent equations, we establish a closedform solution for the Markov model. Second, we calculate the loss probability after FEC recovery as a function of the FEC parameters $(n, k)$.

In the next section, we describe the assumed network model. In sections III and IV] we develop an analytic model of the considered network scenario and calculate the loss probability for multimedia traffic after FEC recovery. An application of the algorithm developed in this paper to a real networking scenario is explained in $\mathrm{V}$. We verify our models numerically in section $\mathrm{VI}$ and conclude in section VII.

\section{NETWORK MODEL AND TERMINOLOGY}

We consider a network model that consists of a multimedia sender and a multimedia receiver, a number of competing 
senders and competing receivers, and a wireless access point. The multimedia sender sends multimedia packets and the competing senders send unspecified packets over wired connections to the AP. The AP forwards all packets over the wireless channel to the multimedia or competing receivers. For simplicity, we assume that all packets are of fixed, equal size.

Packets that arrive at the AP from the senders are either directly sent to the wireless interface of the AP or are queued in a buffer within the AP. The AP buffer has a maximum length $T$ which corresponds to the maximum number of packets that can be buffered. Any packets that arrive when the queue is full, are dropped from the queue. In this paper, we only consider AP-queues that follow a FIFO queueing discipline. The first packet in a queue is sent over the wireless channel if the channel is available. Depending on the wireless technology, a retransmission or a wireless MAC layer FEC scheme might be implemented. Throughout this paper, we assume a discrete, slotted time model. For simplicity, we assume that both the arrival of a packet to the AP from a sender and the successful departure of a packet from the AP over the wireless channel take one time slot. Assuming a Bernoulli model, we define $p_{A}(\leq 1)$ and $p_{C}(\leq 1)$ as the arrival probability at the AP-queue of a multimedia packet and a competing packet, respectively, and set $q_{A}=1-p_{A}, q_{C}=1-p_{C} \cdot p_{D}$ is the probability that in a time slot a packet is sent successfully from the AP queue over the wireless channel and $q_{D}=1-p_{D} \cdot p_{D}$ only represents the rate at which packets leave the AP queue. It does not include the rate at which wireless MAC layer FEC packets or retransmissions of packets are sent over the wireless channel if any of these technologies is implemented in the wireless network. Finally, we abbreviate $\left(\begin{array}{l}a \\ b\end{array}\right)$ as $C_{b}^{a}$. For the analysis, we split a time slot in three sub time slots:

1. Packet arrival phase: Packets are sent from the multimedia sender to the AP with probability $p_{A}$. Competing packets are sent from the competing senders with probability $p_{C}$ to the AP. If packets are sent from both the multimedia and senders, we assume that each of the two packets arrives before the other packet with equal probability $\frac{1}{2}$. The queue length is increased by the number of arriving packets $a \in\{0,1,2\}$.

2. Packet dropping phase : If after the packet arrival phase there are $T+d, d \in\{1,2\}$ packets in the AP-queue, the last $d$ packets are dropped from the queue.

3. Packet sending phase: With probability $p_{D}$ the first packet of the AP-queue is sent over the wireless channel and arrives uncorrupted at the receiver. If the queue is empty, no packet is sent. The queue length is decreased by the number of departing packets $d \in\{0,1\}$.

We define $P_{k}, 0 \leq k \leq T$ as the probability that the AP contains $k$ packets at the end of the packet sending phase of a time slot. In this paper, we will determine the loss probability after FEC recovery for given parameters $p_{A}, p_{C}, p_{D}, T, n$, and $k$.

\section{Steady STATE MOdel of AP QUeUe OCCUPANCY}

In this section, we calculate the steady state probabilities $P_{k}$. As a novelty, we apply the theory of linear recurrent equations to establish an explicit formula for $P_{k}$. We first prove:

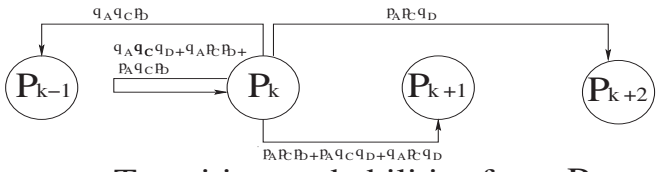

Transition probabilities from $\mathrm{P}_{\mathrm{k}}$

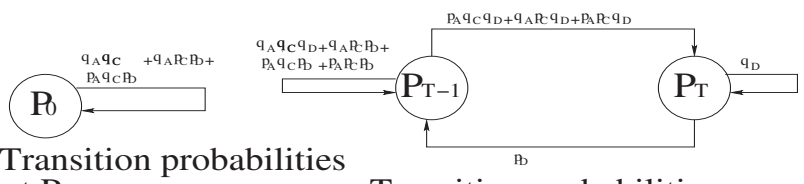

at $\mathrm{P}$

Transition probabilities between $\mathrm{P}_{\mathrm{T}-1}$ and $\mathrm{P}_{\mathrm{T}}$

Fig. 1. The transition probabilities for the AP queue occupancy Theorem 1:

$$
\begin{aligned}
P_{0} & =\frac{1}{1+\sum_{k=1}^{T-1} \frac{A^{k}}{C^{k}} B_{k}+\frac{Y}{p_{D}}}, \\
P_{k} & =P_{0} \frac{A^{k}}{C^{k}} B_{k}, \quad 1 \leq k \leq T-1, \\
P_{T} & =\frac{P_{0}}{p_{D}} Y,
\end{aligned}
$$

where

$$
\begin{aligned}
B_{k}= & \sum_{l=0}^{\frac{k}{2}}\left(\frac{B C}{A^{2}}\right)^{l} C_{l}^{k-l}, 1 \leq k \leq T-1 \\
A= & p_{A} p_{C}+q_{A} p_{C} q_{D}+p_{A} q_{C} q_{D} \\
B= & p_{A} p_{C} q_{D}, \quad C=q_{A} q_{C} p_{D} \\
Y= & \left(1+C-p_{D}-q_{A} q_{C} q_{D}\right) \frac{A^{T-1}}{C^{T-1}} B_{T-1} \\
& -(A-B) \frac{A^{T-1}}{C^{T-2}} B_{T-2}-B \frac{A^{T-3}}{C^{T-3}} B_{T-3}
\end{aligned}
$$

From the network model in section II and Fig. 1, we see:

$$
\begin{aligned}
P_{0} & =P_{0}\left(q_{A} q_{C}+q_{A} p_{C} p_{D}+p_{A} q_{C} p_{D}\right)+P_{1} q_{A} q_{C} p_{D},(8) \\
P_{1} & =P_{1}\left(q_{A} q_{C} q_{D}+q_{A} p_{C} p_{D}+p_{A} q_{C} p_{D}\right) \\
& +P_{0}\left(p_{A} p_{C} p_{D}+p_{A} q_{C} q_{D}+q_{A} p_{C} q_{D}\right)+P_{2} q_{A} q_{C} p_{D}, \\
P_{k} & =P_{k}\left(q_{A} q_{C} q_{D}+q_{A} p_{C} p_{D}+p_{A} q_{C} p_{D}\right) \\
& +P_{k-1}\left(p_{A} p_{C} p_{D}+p_{A} q_{C} q_{D}+q_{A} p_{C} q_{D}\right) \\
& +P_{k+1} q_{A} q_{C} p_{D}+P_{k-2} p_{A} p_{C} q_{D}, 2 \leq k \leq T-2, \\
P_{T-1} & =P_{T-1}\left(q_{A} q_{C} q_{D}+q_{A} p_{C} p_{D}+p_{A} q_{C} p_{D}+p_{A} p_{C} p_{D}\right) \\
& +P_{T} p_{D}+P_{T-2}\left(p_{A} p_{C} p_{D}+p_{A} q_{C} q_{D}+q_{A} p_{C} q_{D}\right) \\
& +P_{T-3} p_{A} p_{C} q_{D}, \\
P_{T} & =P_{T} q_{D}+P_{T-1}\left(p_{A} q_{C} q_{D}+q_{A} p_{C} q_{D}+p_{A} p_{C} q_{D}\right) \\
& +P_{T-2} p_{A} p_{C} q_{D} .
\end{aligned}
$$

Using the definitions (5) - (6), we rewrite $(\underline{8})-(9)$ :

$$
\begin{aligned}
C P_{1}= & P_{0} A \\
C P_{2} & =P_{1}(A+C)-P_{0}(A-B), \\
P_{k+3} C= & P_{k+2}(A+C)-P_{k+1}(A-B)-P_{k} B, \\
& 0 \leq k \leq T-4, \\
P_{T} p_{D}= & P_{T-1}\left(1+C-p_{D}-q_{A} q_{C} q_{D}\right) \\
- & P_{T-2}(A-B)-P_{T-3} B .
\end{aligned}
$$

The relations (11), (12), and (13) imply (2) for $k=1,2,3$. Using induction and (13), we show that (2) holds for $k+3, k+$ 
$3<T$, provided it holds for $k, k+1$, and $k+2$. To prove this claim, we insert the expression (2) in (13), and now have to show that:

$$
\begin{aligned}
C \frac{A^{k+3}}{C^{k+3}} B_{k+3} & =(A+C) \frac{A^{k+2}}{C^{k+2}} B_{k+2}-(A-B) \frac{A^{k+1}}{C^{k+1}} B_{k+1} \\
& -B \frac{A^{k}}{C^{k}} B_{k},
\end{aligned}
$$

which is equivalent to

$$
\begin{aligned}
A^{3} B_{k+3} & =A^{3} B_{k+2}+A^{2} C B_{k+2}-A^{2} C B_{k+1} \\
& +A B C B_{k+1}-B C^{2} B_{k+1},
\end{aligned}
$$

which we write as $X_{1}=X_{2}+X_{3}-X_{4}+X_{5}-X_{6}$. We recall that $C_{k}^{n+1}=C_{k}^{n}+C_{k-1}^{n}, C_{0}^{n}=1$. Thus, from (4)

$$
\begin{aligned}
& X_{1}-X_{2}-X_{5} \\
= & A^{3} \sum_{l=0}^{\frac{k+3}{2}}\left(\frac{B C}{A^{2}}\right)^{l} C_{l}^{k+3-l}-A^{3} \sum_{l=0}^{\frac{k+2}{2}}\left(\frac{B C}{A^{2}}\right)^{l} C_{l}^{k+2-l} \\
& -A^{3} \sum_{l=1}^{\frac{k+3}{2}}\left(\frac{B C}{A^{2}}\right)^{l} C_{l-1}^{k+1-(l-1)} \\
= & A^{3} \sum_{l=1}^{\frac{k+2}{2}}\left(\frac{B C}{A^{2}}\right)^{l}\left(C_{l}^{k+3-l}-C_{l}^{k+2-l}-C_{l-1}^{k+2-l}\right) \\
& +A^{3}\left(C_{0}^{k+3}-C_{0}^{k+2}\right) \\
& +A^{3} \sum_{\frac{k+2}{2}<l \leq \frac{k+3}{2}}\left(\frac{B C}{A^{2}}\right)^{l-1}\left(C_{l}^{k+3-l}-C_{l-1}^{k+1-(l-1)}\right) \\
= & 0 . \quad 16)
\end{aligned}
$$

In the same way, we see $X_{3}-X_{4}-X_{6}=0$, which together with (16) proves (15). For $k=T$, (3) follows from (2) for $k<T$, and (14). (1) follows from (2), (3), and the fact that $\sum_{k=0}^{T} P_{k}=1$.

Now, we simplify the expressions derived for $P_{k}$ in Theorem 1. We extend the definition of the $B_{k}$ in (4) by defining $B_{0}$ as $B_{0}=1$. First, we show:

$$
B_{k+1}=B_{k}+\left(\frac{B C}{A^{2}}\right) B_{k-1}, k \geq 1 .
$$

For the proof, we show that

$$
\begin{aligned}
& C_{0}^{k+1}+\sum_{l=1}^{\frac{k}{2}}\left(\frac{B C}{A^{2}}\right)^{l} C_{l}^{k+1-l}+\sum_{\frac{k}{2}<l \leq \frac{k+1}{2}}\left(\frac{B C}{A^{2}}\right)^{l} C_{l}^{k+1-l} \\
& =C_{0}^{k}+\sum_{l=1}^{\frac{k}{2}}\left(\frac{B C}{A^{2}}\right)^{l} C_{l}^{k-l}+\sum_{l=1}^{\frac{k}{2}}\left(\frac{B C}{A^{2}}\right)^{l} C_{l-1}^{k-l} \\
& +\sum_{\frac{k}{2}<l \leq \frac{k+1}{2}}\left(\frac{B C}{A^{2}}\right)^{l} C_{l-1}^{k-l},
\end{aligned}
$$

which we write as $Y_{1}+Y_{2}+Y_{3}=Y_{4}+Y_{5}+Y_{6}+Y_{7}$. Arguing as above, we see $Y_{2}=Y_{5}+Y_{6}, Y_{1}=Y_{4}=1$, and $Y_{3}=Y_{7} \in\{0,1\}$.

We recall some facts from the theory of linear recurrent equations: For a series defined as $x_{0}=x_{1}=1$ and $x_{n}=$ $a x_{n-1}+b x_{n-2}, n \geq 2$ where $a, b \in \mathbb{R}^{+}$, there is,

$$
x_{n}=\frac{1}{\sqrt{a^{2}+4 b}}\left[(1-\beta) \alpha^{n}+(\alpha-1) \beta^{n}\right],
$$

where $\alpha$ and $\beta$ are the roots of the equation $x^{2}=a x+b$, i.e., (15) $\alpha=\frac{1}{2}\left(a+\sqrt{a^{2}+4 b}\right), \beta=\frac{1}{2}\left(a-\sqrt{a^{2}+4 b}\right)$. This follows directly for $n=0,1$ and by induction for $n \geq 2$.

From (17), (18), and the fact that for the $B_{k}$, there is $1-\beta=\alpha$, we see that $B_{k}=B_{k}^{*}$, where

$$
\begin{aligned}
B_{k}^{*}= & \frac{\left(1+K_{A B C}\right)^{k+1}-\left(1-K_{A B C}\right)^{k+1}}{2^{k+1} K_{A B C}}, \\
& K_{A B C}=\sqrt{1+4 \frac{B C}{A^{2}}}, 0 \leq k \leq T-1 .
\end{aligned}
$$

We use relation (19) to calculate $D:=\sum_{k=1}^{T-1} \frac{A^{k}}{C^{k}} B_{k}^{*}$ as follows:

$$
\begin{aligned}
D & =\frac{A}{C K_{A B C}}\left[\left(\frac{1+K_{A B C}}{2}\right)^{2} \sum_{k=0}^{T-2} \frac{\left(A\left(1+K_{A B C}\right)\right)^{k}}{(2 C)^{k}}\right. \\
& \left.-\left(\frac{1-K_{A B C}}{2}\right)^{2} \sum_{k=0}^{T-2} \frac{\left(A\left(1-K_{A B C}\right)\right)^{k}}{(2 C)^{k}}\right] \\
& =\frac{A}{C K_{A B C}}\left[\left(\frac{1+K_{A B C}}{2}\right)^{2} \frac{\frac{\left(A\left(1+K_{A B C}\right)\right)^{T-1}}{(2 C)^{T-1}}-1}{\frac{A\left(1+K_{A B C}\right)}{2 C}-1}\right. \\
& \left.-\left(\frac{1-K_{A B C}}{2}\right)^{2} \frac{\frac{\left(A\left(1-K_{A B C}\right)\right)^{T-1}}{(2 C)^{T-1}}-1}{\frac{A\left(1-K_{A B C}\right)}{2 C}-1}\right] .
\end{aligned}
$$

From Theorem 1, (19), and (20), we obtain Theorem 2 which reduces the complexity of the calculation of the $P_{k}$ in Th. 1 . Here $Y^{*}$ is defined as $Y$ in (7) with $B_{k}$ replaced by $B_{k}^{*}$.

Theorem 2:

$$
\begin{aligned}
P_{0} & =\frac{1}{1+D+\frac{Y}{p_{D}}}, \\
P_{k} & =P_{0} \frac{A^{k}}{C^{k}} B_{k}^{*}, \quad 1 \leq k \leq T-1, \\
P_{T} & =\frac{P_{0}}{p_{D}} Y^{*} .
\end{aligned}
$$

\section{PACKET LOSS PROBABILITY AFTER FEC RECOVERY}

We first calculate the probability $P_{\text {loss }}$ that a multimedia or FEC packet arriving at the AP in the packet arrival phase is dropped in the packet dropping phase of the same time slot. This happens if at the beginning of a time slot:

- There are $T-1$ packets in the queue, and a competing packet arrives before the multimedia at the queue.

- There are $T$ packets in the queue.

Consequently,

$$
P_{\text {loss }}=\frac{1}{2} P_{T-1} p_{C}+P_{T}
$$

Based on our model (sec. II), the factor $\frac{1}{2}$ expresses the probability that if in a time slot packets are sent from both the multimedia and the competing sender, the competing packet arrives before the multimedia packet. We note that to apply 
Theorem 2 to calculate $P_{T-1}$ and $P_{T}$ in (21), $p_{A}$ is increased to $p_{A} \frac{n}{k}$ to account for the FEC overhead.

Arguing as in [7], we now calculate $p_{i}(k, n)$ as the probability to lose $i$ multimedia and at least $n-k-i+1$ FEC packets at the receiver and $E_{l o s s}$ as the probability to lose an individual multimedia packet after FEC recovery as follows:

$$
\begin{aligned}
p_{i}(k, n)= & C_{i}^{k} P_{\text {loss }}^{i} Q_{\text {loss }}^{k-i} \sum_{m=\max (0, n-k-i+1)}^{n-k} C_{m}^{n-k} \\
& \times P_{\text {loss }}^{m} Q_{\text {loss }}^{n-k-m}, \\
E_{\text {loss }}= & \frac{1}{k} \sum_{i=1}^{k} i \times p_{i}(k, n) .
\end{aligned}
$$

Using Theorem 2, (21), and (22), we see that if we compute the values of $C_{b}^{a}$ forehand, the complexity of the calculation of $E_{\text {loss }}$ is $O(k n)$. This is less than the complexity $O\left(n^{2} k^{2}+\right.$ $\left.T n k^{2}\right)$ of the (exact) method in [4].

\section{APPLICATION SCENARIO}

In a real application, the multimedia sender must know all the relevant input parameters to calculate $P_{l o s s}(21)$ and $E_{l o s s}$ (22). The multimedia sender knows the multimedia arrival rate $p_{A}$ and the parameters $(n, k)$. The maximum queue length $T$ is a static value which at the time of network configuration can be communicated to the sender. The value of $p_{D}$ can be derived by deploying a specialized wireless MAC layer measurement software, which has been developed by the authors, at the receiver. However, the arrival rate of the competing traffic $p_{C}$ is in general not available to the multimedia sender.

In this section, we propose a two-step procedure to calculate the values $P_{\text {Loss }}$ and $E_{\text {loss }}$ when the actual value of $p_{C}$ is not known to the multimedia sender. First, for given values of $p_{A}, p_{D}, P_{\text {Loss }}$ if no FEC protection is applied and for given values of $p_{A}, p_{D}, n, k, E_{l o s s}$ if FEC protection is applied, the equations (21) and (22) are used to determine the actual value of $p_{C}$. This procedure is explained in detail in the next paragraph. Second, in order to determine an optimal FEC parameter set $(n, k)$, the determined value of $p_{C}$ is used as an input parameter to the expression (21).

The derivation of (21) and Th. 2 shows that for given values of $p_{A}, p_{D}$, and $T$ the formula (21) establishes a one-to-one and monotonic mapping between $p_{C}$ and $P_{l o s s}$, i.e., for a given value of $P_{\text {loss }}$ there is one possible choice of $p_{C}$. Similar, for given $p_{A}, p_{D}, T$, and $n, k$, the relation (22) establishes a oneto-one and monotonic mapping between $p_{C}$ and $E_{l o s s}$.

The multimedia server knows the actual values of $p_{A}, n$, and $k$, and can obtain $P_{\text {Loss }}$ or $E_{\text {loss }}$ from the multimedia receiver. In view of the monotonic relation between $p_{C}$ and $P_{\text {loss }}$ or $E_{\text {loss }}$, the multimedia server now determines the actual value of $p_{C}$ as follows: It performs an iterative search over possible values $p_{C}$ that uses a binary chop strategy to find the actual value of $p_{C}$ with a predefined accuracy.

In practice, the feasible choices of $n$ and $k$ will be limited by the bandwidth and latency overhead introduced by the FEC code. The procedure described in this paragraph can be used to find the parameter set $n$ and $k$ among all feasible choices of $n$ and $k$ that minimizes the loss probability after FEC recovery.

\section{NUMERICAL RESULTS}

In this section, we present simulation results that verify the accuracy of the expressions (21) and (22). We used the $\mathrm{C}$ programming language to build a model of the network model and simulated 1000000 consecutive time slots. Fig. 2 shows the change of the multimedia packet recovery rate after FEC recovery as a function of the FEC parameter $k$. The simulations show that the analytic model fits exactly the simulation results, i.e., the difference between analytical and simulation results is within the expected statistical variance of the simulations. The difference is so fine that the respective values cannot be distinguished in a graph.

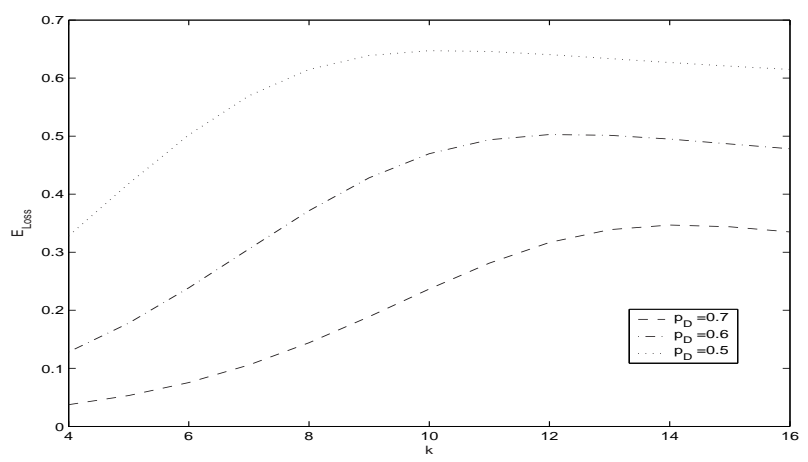

Fig. 2. The loss probability after FEC recovery for changing $k$ with $\mathrm{T}=200$, $p_{A}=0.2, p_{C}=0.5, \mathrm{n}=17$.

\section{CONCLUSIONS}

This paper presents an algorithm to determine the optimal choice of FEC parameters $(n, k)$ to protect a multimedia stream sent over a wireless network from packet losses due to packet dropping at the queue of the wireless access point. We develop a mathematical model of the wireless network that takes into account the exact arrival pattern of the multimedia stream, the accumulative arrival rate of all other traffic arriving at the wireless access point, the loss model of the wireless channel, and the queue size of the wireless access point.

The theory of linear recurrent equations is applied to establish a closed form solution of the steady state Markov model for the buffer occupancy. We propose a deployment scenario for the algorithm and verify the effectiveness of the algorithm via network simulations.

\section{REFERENCES}

[1] Adoul, J.-P.A.; Error intervals and cluster density in channel modeling, IEEE Trans. Inform. Theory, vol. 20, 125129, Jan. 1974.

[2] Blahut, R., Theory and Practice of Error control codes, Addison-Wesley, 1993.

[3] Hayasaka, M.; Loyola, L.; Miki, T., Packet/cell loss recovery using variable FEC matrix for real time transport services over best effort networks, 1119- 1123, Vol.3, IEEE APCC 2003.

[4] Ait-Hellal, O.; Altman, E.; Jean-Marie, A.; Kurkova, I.; On loss probabilities in presence of redundant packets and several traffic sources, Performance Evaluation, 36 - 37 (1999), 485 - 518.

[5] Johanson M.; Adaptive forward error correction for real-time internet video, Proc. of the 13th Packet Video Workshop. 2003.

[6] Frossard, P., FEC Performances in Multimedia Streaming Pascal, IEEE Comm. Letters, vol.5, no 3, March 2001, 122-124.

[7] Jurca, D.; Frossard, P., Optimal FEC Rate for Media Streaming in Active Networks, IEEE ICME 2004, Taipei.

[8] Libman, L.; Orda, A., Optimal FEC Strategies in Connections with Large Delay-Bandwidth Products, IEEE ICC 2004.

[9] Rizzo, L.; Effective erasure codes for reliable computer communication protocols, ACM Comp. Comm. Review, vol. 27, no. 2, 2436, Apr. 1997. 